\title{
Eine tägliche Herausforderung im Büroalltag: Die elektronische Rechnung
}

Die elektronische Rechnung ist im heutigen Büroalltag nicht mehr wegzudenken. Sie löst immer mehr die klassische Papierrechnung ab. Viele Unternehmer empfinden diese Situation nicht nur als praktisch, sondern sehen hierin nicht selten ein Potenzial zur Kostenersparnis und somit zur Gewinnsteigerung.

Dass der Einsatz der E-Rechnung - wie die elektronische Rechnung auch genannt wird - im Tagesgeschehen nicht nur positive Seiten, sondern auch Herausforderungen mit sich bringt, soll unter anderem mit Hilfe dieses Essentials aufgezeigt werden. Neben der allgemeinen Darstellung des inländischen Umsatzsteuer-Systems werden wesentliche gesetzliche Grundlagen und die im November 2014 erschienenen GoBD (= Grundsätze zur ordnungsmäßigen Führung und Aufbewahrung von Büchern, Aufzeichnungen und Unterlagen in elektronischer Form sowie zum Datenzugriff) [1] behandelt, welche beim Einsatz von elektronischen Rechnungen unbedingt zu beachten sind. Besonderheiten bei Fahrausweisen oder Kleinbetragsrechnungen sind ebenfalls Bestandteil dieses Essentials sowie eine kritische Betrachtung bzgl. des Einsatzes von elektronischen Rechnungen im Büroalltag. Wichtige Links, die Sie, liebe Leserinnen und Leser, bei Ihrer täglichen Arbeit im Büro unterstützen sollen, bildet den Abschluss dieses Essentials.

Ein Anspruch auf Vollständigkeit kann aufgrund der Komplexität dieses Themas leider nicht erhoben werden.

Als Autorin freue ich mich sehr auf Ihr Feedback zu diesem Essential. Gerne können Sie mir Ihre Kommentare, Ideen und Hinweise zu diesem komplexen Themengebiet per Mail (office@karin-nickenig.com) mitteilen. Ich bin gespannt!! Vielen Dank! 